\title{
FDG PET-CT: Need for Vigilance in Patients Treated with Bleomycin
}

\begin{abstract}
Bleomycin-induced interstitial lung disease is not a rare complication of treatment and may prove to be fatal if not suspected early in its course. As FDG PET-CT has become an essential clinical tool for interim and end-of-treatment response assessment in oncology, it is important that radiologists/ nuclear medicine specialists be aware of the features of this potentially treatable condition so that treatment can be instigated during an early reversible phase before pulmonary damage has occurred. Herein, we report two cases of bleomycin toxicity featuring different patterns on the treatmentresponse assessment FDG PET-CT scan.
\end{abstract}

Key words: bleomycin, FDG, lung toxicity, PET-CT

\section{Introduction}

More than 450 medications are known to cause lung toxicity with the most common form being interstitial lung disease (ILD). ${ }^{[1]}$ Among chemotherapeutic drugs, bleomycin is the most commonly implicated in ILD. ${ }^{[1]}$

The mechanism of bleomycin-induced ILD is not entirely clear, but likely attributed to the combination of direct oxidative damage and indirect immune cells-mediated injury. ${ }^{[2]}$ Bleomycin is inactivated in vivo by the enzyme bleomycin hydrolase, which has lower activity in the skin and lungs, rendering these organs more susceptible in oxygen radical effects. ${ }^{[2]}$ From the limited number of histopathology findings, it is known that ILD begins with the accumulation of inflammatory cells in lung interstitium, causing progressive destruction of lung parenchyma, which is followed by healing and scarring, leading to irreversible fibrotic change. ${ }^{[3]}$

Although the exact frequency of bleomycininduced pulmonary toxicity is not known, as the diagnosis is usually presumptive, it is often quoted as approximately $10 \%$ of all patients receiving the drug with a mortality rate of $3-5 \%$ of those affected. ${ }^{[4]}$

The risk of developing bleomycininduced ILD is influenced mainly by the cumulative drug dose, the disease burden in lungs, and the concurrent use of other

This is an open access article distributed under the terms of the Creative Commons Attribution-NonCommercial-ShareAlike 3.0 License, which allows others to remix, tweak, and build upon the work non-commercially, as long as the author is credited and the new creations are licensed under the identical terms.

For reprints contact: reprints@medknow.com lung-toxic chemotherapeutic agents or radiotherapy. ${ }^{[4]}$

Bleomycin-induced ILD may occur during therapy, subacutely between 1 and 6 months after treatment or more than 6 months following its administration. ${ }^{[4]}$ Diagnosis is challenging because of the absence of symptoms or the non-specific nature of clinical features and radiologic or laboratory findings. ${ }^{[4]}$ Particularly, in patients with compromised immune function, differential of infection is always possible.

As imaging investigations have become very prevalent in the management of oncologic patients, radiologists/nuclear medicine specialists must be vigilant, as these may provide the only early sign, instigating the diagnosis of this potentially life-threatening condition.

High-resolution computed tomography (HRCT) is the most sensitive modality for detecting parenchymal abnormalities. HRCT findings of the drug-induced ILD range from scattered or diffuse ground-glass opacities or consolidation predominantly of peripheral and basal distribution. As the interestitium destruction progresses and fibrosis develops, more prominent reticular appearances, traction bronchiectasis, and honeycombing predominate. ${ }^{[5]}$

FDG PET-CT has established its role for the treatment response assessment in

How to cite this article: Paschali AN, Gnanasegaran G, Cook GJ. FDG PET-CT: Need for vigilance in patients treated with bleomycin. Indian J Nucl Med 2017;32:122-4.

\section{Anna N Paschali, Gopinath Gnanasegaran', Gary $\mathrm{J} \mathrm{Cook}^{2}$}

Theagenion Cancer Hospital, Thessaloniki, Greece, ${ }^{\text {I}}$ Royal Free London NHS Foundation Trust, ${ }^{2} \mathrm{KCL}$ Division of Imaging Sciences \& Biomedical Engineering, London, $U K$

Address for correspondence: Dr. Anna N. Paschali, Theagenion Cancer Hospital, Alexandrou Simeonidi,

Thessaloniki, Greece.

E-mail: a_pashali@yahoo.gr

Access this article online Website: www.indjsp.org DOI: 10.4103/0972-3919.202242 Quick Response Code:

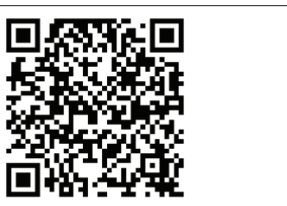




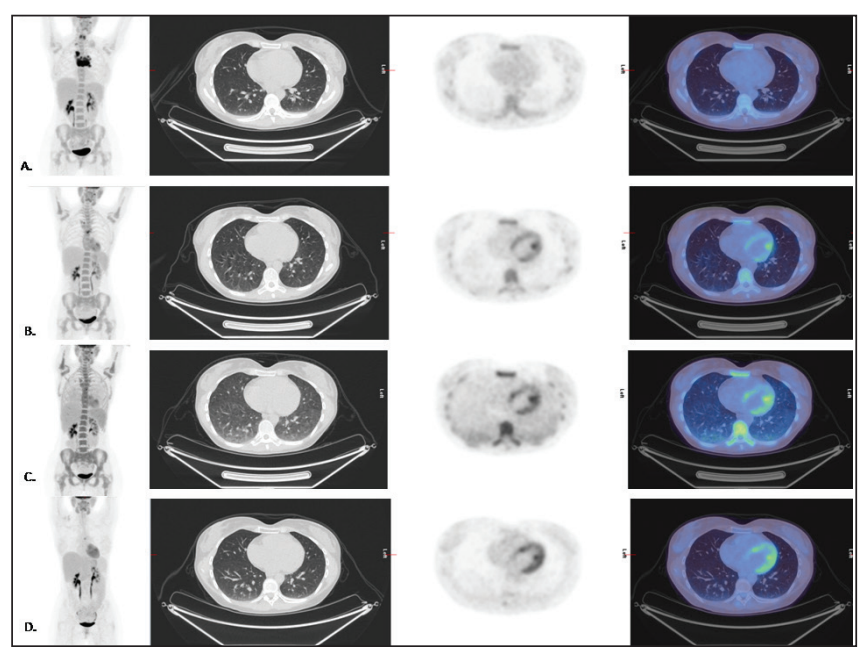

Figure 1: MIP and Axial CT, FDG PET, and fused PET-CT images in a patient with Hodgkin's disease at (a) Baseline, (b) Post-2 ABVD cycles, (c) Post-4 ABVD cycles, and (d) end of treatment (4ABVD + 2AVD) cycles response assessment PET/CT scans. (a) Stage 2B bulky HD in the mediastinum. (b) Partial response to chemotherapy with a small remnant mediastinal focus (Deauville 4). (c) Further response to chemotherapy with a remaining small mediastinal focus of increased uptake (Deauville 4). The lungs demonstrate diffusely increased tracer uptake with underlying areas of increased parenchymal attenuation at the mid and lower zones. (d) End-of-treatment response assessment: there has been a response to treatment with a further reduction in the size of the residual anterior mediastinal mass and reduction in the remaining focus of increased activity (however this remained Deauville 4). There has been resolution of the previous diffuse tracer uptake in the lungs, and on the CT component of the study, the lungs now appear clear following cessation of bleomycin and treatment with corticosteroids.

many malignancies. Particularly in lymphomas, there is an increasing use of mid-treatment response assessment scans within this clinical context. As FDG is not a tumor-specific tracer, new lung lesions, identified on the PET only or the CT component of the study, are commonly seen during the mid- or end-of-treatment PET-CT scans. These new lesions are usually considered of infective origin, in the context of non-pulmonary involvement at the baseline scan and metabolic response of the other disease sites. ${ }^{[6]}$ However, in patients receiving or having received bleomycin, new lung parenchymal abnormalities on PET-CT, as those described in the following paragraphs, should also raise the possibility of lung toxicity and the referring oncologist should be alerted.

\section{Case Report}

We report two cases of bleomycin toxicity featuring different patterns on the treatment-response assessment FDG PET-CT scan.

Case 1: A 40-year-old female, with stage 2B bulky Hodgkin's disease (HD) in the mediastinum [Figure 1], was treated with doxorubicin, bleomycin, vinblastine, and dacarbazine (ABVD). Interim FDG PET-CT scans after two and four cycles of ABVD showed persistent uptake within the mediastinal mass (Deauville score 4). ${ }^{[7]}$ Furthermore, in the post-4-cycle PET scan, there was diffusely increased

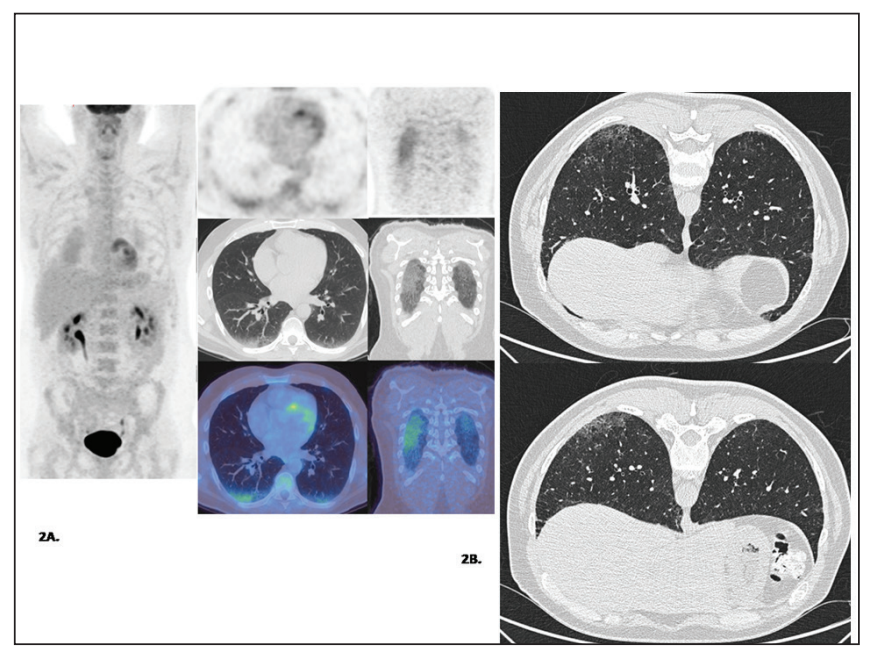

Figure 2: Patient with stage II seminoma following three cycles of BEP chemotherapy. (a) Post-treatment MIP FDG PET, axial and coronal PET, CT and fused PET/CT images demonstrate increased tracer uptake at both lung bases corresponding to ground glass opacity on CT (R>L). (b) Highresolution $\mathrm{CT}$ Thorax (prone scan): There is persistent subtle ground glass change seen at the right lung base with minimal ground glass changes at the left lung base.

tracer uptake within the lungs, with underlying areas of increased parenchymal attenuation on the low-dose CT component in the mid and lower zones. The PET appearances raised the possibility of pulmonary toxicity and the referring clinician was alerted. The patient at that phase was asymptomatic and the possibility of infection was considered less likely. A diagnosis of bleomycin pulmonary toxicity was made and optimal treatment initiated. The patient remained asymptomatic, and at the end of treatment, after two more cycles of AVD, the lung appearances normalized. A small focus of increased uptake (Deauville score 4) persisted within the mediastinum and the patient was referred for radiotherapy treatment.

Case 2: A 45-year-old man with stage II seminoma, who had undergone left orchidectomy and completed three cycles of chemotherapy (bleomycin, etoposide, and cisplatin), had a post-treatment PET-CT 6 weeks after completion of chemotherapy. The scan showed moderately increased tracer uptake at both lung bases corresponding to ground glass opacities on $\mathrm{CT}$, more prominent on the right lung [Figure 2a], which raised the possibility of bleomycin toxicity. The patient at that phase was asymptomatic and the possibility of infection was considered less likely. On the subsequent thoracic HRCT (prone scan), there is persistent subtle ground glass change seen at the right lung base with minimal ground glass changes at the left lung base [Figure 2b]. The appearances and clinical features were considered to be due to bleomycin toxicity and treatment was commenced.

\section{Discussion}

These cases document that FDG PET can detect metabolic changes at a phase where the clinical symptoms are 
ambiguous or absent with no pathognomonic changes evident on the low-dose CT component of the study. Early commencement of optimal treatment (e.g., cessation of bleomycin and starting corticosteroids) is of critical importance, as bleomycin-induced ILD is reversible only in the inflammatory phase and not in the fibrotic stage.

FDG uptake in bleomycin-induced ILD has previously been reported in case reports in the literature mainly in $\mathrm{HD}^{[8-11]}$ and in one seminoma case. ${ }^{[12]}$ In a retrospective study in lymphoma patients undergoing FDG PET for the evaluation of chemotherapy response, $2.2 \%$ of cases were identified as probable drug toxicity, showing diffuse and sub-pleural FDG accumulation corresponding to scattered or diffuse ground glass opacities on the $\mathrm{CT}$. The majority of the patients were asymptomatic at the time of the PET scan. ${ }^{[10]}$ Buchler et al. also highlighted the significance of FDG PET for deciding whether to continue or discontinue treatment with anti-inflammatory agents, on the grounds that PET-CT, in contrast to lung function tests and conventional $\mathrm{CT}$, has the potential to distinguish between active inflammation and permanent lung damage. ${ }^{[1]}$

\section{Financial support and sponsorship}

Nil

\section{Conflicts of interest}

There are no conflicts of interest

\section{References}

1. Schwaiblmair M, Behr W, Haeckel T, Märkl B, Foerg W, Berghaus T. Drug induced interstitial lung disease. Open Respir Med J 2012;6:63-74.

2. Chandler DB. Possible mechanisms of bleomycin-induced fibrosis. Clin Chest Med 1990;11:21.
3. Jules-Elysee K, White DA. Bleomycin-induced pulmonary toxicity. Clin Chest Med 1990;11:1.

4. Bradley B, Branley HM, Egan JJ, Greaves MS, Hansell DM, Harrison NK, et al. Interstitial lung disease guideline: the British Thoracic Society in collaboration with the Thoracic Society of Australia and New Zealand and the Irish Thoracic Society. Thorax 2008;63:1-58.

5. Padley SP, Adler B, Hansell DM, Müller NL. High-resolution computed tomography of drug-induced lung disease. Clin Radiol 1992;46:232-6.

6. Juweid ME, Stroobants S, Hoekstra OS, Mottaghy FM, Dietlein M, Guermazi A, et al. Use of positron emission tomography for response assessment of lymphoma: consensus of the Imaging Subcommitee of International Harmonization Project in Lymphoma. J Clin Oncol 2007;25:571-8.

7. Cheson BD, Fisher RI, Barrington SF, Cavalli F, Schwartz LH, Zucca E, et al. Recommendations for initial evaluation, staging, and response assessment of Hodgkin and nonHodgkin lymphoma: the Lugano classification. J Clin Oncol 2014;32:3059-68.

8. Kirsch J, Arrossi AV, Yoon JK, Wu G, Neumann DR. FDG positron emission tomography/computerized tomography features of bleomycin-induced pneumonitis. J Thorac Imaging 2006;21:228-30

9. Batra S, Vaid AK, Bhargava R, Gupta S. Lungs on fire. BMJ Case Rep 2013;27.

10. Kazama T, Faria SC, Uchida Y, Macapinlac HA. Pulmonary drug toxicity: FDG-PET findings in patients with lymphoma. Ann Nucl Med 2008;22:111-4.

11. Buchler T, Bomanji J, Lee SM. FDG-PET in bleomycin-induced pneumonitis following ABVD chemotherapy for Hodgkin's disease-a useful tool for monitoring pulmonary toxicity and disease activity. Haematologica 2007;92:120-1.

12. von Rohr L, Klaeser B, Joerger M, Kluckert T, Cerny $\mathrm{T}$, Gillessen S. Increased pulmonary FDG uptake in bleomycinassociated pneumonitis. Onkologie 2007;30:320-3. 